\section{The two faces of autophagy and the pathological underestimation of DCIS}

\author{
Ke-Da Yu and Zhi-Ming Shao
}

We read with great interest the Opinion article (What is the malignant nature of human ductal carcinoma in situ? Nature Rev. Cancer 11, 68-75 (2011)) by Espina and Liotta ${ }^{1}$, in which the authors present the current knowledge on potential survival mechanisms of ductal carcinoma in situ (DCIS) cells, propose a novel strategy for killing DCIS cells and introduce an ongoing neoadjuvant therapy trial. However, several important issues related to the antiautophagy therapy for DCIS need further discussion.

Regarding breast cancer, in invasive ductal carcinoma (IDC) cells or DCIS microinvasive cells (FIG. 1; solid square), which have a sufficient supplement of oxygen and nutrients that have diffused from nearby blood vessels through the extracellular matrix, autophagy is not the main mechanism for cell survival, and antiautophagy treatments that only lead to p62 accumulation might enhance cancer progression $^{2-4}$. By contrast, in pure DCIS cells (FIC. 1; dashed square), autophagy is a vital pathway for cell survival, and the reasons for using anti-autophagy therapies in treating DCIS were clearly discussed by Espina and Liotta ${ }^{1}$.

As it seems that we need to avoid antiautophagy treatment in invasive breast cancer to prevent cancer progression, the question is therefore, how can we determine that a patient has only DCIS rather than an invasive component of, or even frank, IDC? DCIS with an invasive component is common. We have previously shown that among 550 DCIS cases, $49.3 \%$ were pure DCIS, $12.2 \%$ were DCIS with microinvasion and $38.5 \%$ had an invasion component

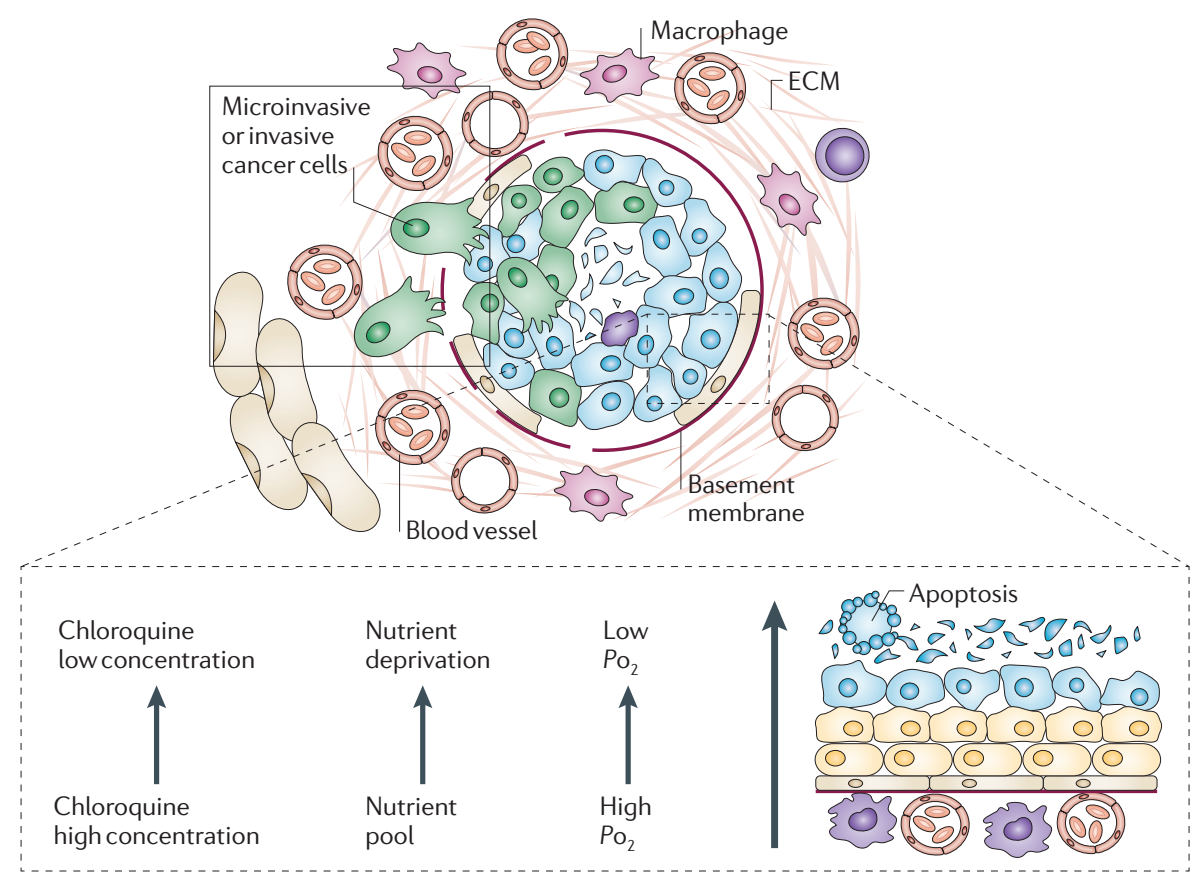

Figure 1 | Differing autophagy effects and survival mechanisms of ductal carcinoma in situ (DCIS) and microinvasive or invasive ductal carcinoma (IDC). In IDC cells that have easy access to blood vessels and have a sufficient supply of oxygen and nutrients, autophagy is not the main cell survival mechanism under normal conditions. When the anti-autophagy agent is administered, not only is the degradation of toxic material prevented, but the accumulation of p62 following autophagy inhibition could also promote tumorigenesis. In DCIS cells, autophagy is an important pathway that is activated to promote survival in the face of hypoxic and nutrient stress. Autophagy-competent cells would have a better chance of survival. ECM, extracellular matrix.

that exceeded microinvasion ${ }^{5}$. Many DCIS cases that were initially diagnosed by coreneedle biopsy (CNB) have proved to be IDC. Compared with open surgical biopsy, CNB is reported to be safer and more costeffective; however, it has an inherent problem of sampling error, which contributes to a significant DCIS underestimation rate of about $26 \%{ }^{6}$. High-grade lesions at CNB, and lesion size of larger than $20 \mathrm{~mm}$ at imaging, are significantly associated with greater odds of invasive carcinoma after definitive surgery ${ }^{6}$. Regarding the neoadjuvant preventing invasive neoplasia with chloroquine (PINC) trial, which examines the safety and effectiveness of chloroquine administration to patients with $\mathrm{DCIS}^{1}$, a certain proportion of patients diagnosed with DCIS using $\mathrm{CNB}$ will probably have an invasive component. Those underestimated cases are not good candidates for treatment using the antiautophagy strategy.

Furthermore, as DCIS is confined to a duct without a blood supply, chloroquine, like oxygen and nutrients, has no direct way of reaching the autophagy-competent cancer cells. Chloroquine will need to diffuse from the blood vessels into the surrounding tissue, across the basement membrane and into the ducts affected by DCIS to reach most of the autophagy-dependent cells that are located furthest from the basement membrane (FIG. 1). Whether chloroquine can successfully reach the intraductal niche at a therapeutic concentration is questionable.

Ke-Da Yu and Zhi-Ming Shao are at the Department of Breast Surgery, Cancer Center and Cancer Institute, and the Department of Oncology, Shanghai Medical College, Fudan University, Shanghai 200032, China, as well as the Institutes of Biomedical Science, Fudan University, Shanghai 200032, China. Correspondence to Z.-M.S. e-mail: zhimingshao@yahoo.com doi:10.1038/nrc2950-c1

1. Espina, V. \& Liotta, L. A. What is the malignant nature of human ductal carcinoma in situ? Nature Rev. Cancer 11, 68-75 (2011).

2. Mathew, R. \& White, E. Autophagy in tumorigenesis and energy metabolism: friend by day, foe by night. Curr. Opin. Genet. Dev. 21, 113-119 (2011).

3. Chen, N. \& Karantza, V. Autophagy as a therapeutic target in cancer. Cancer Biol. Ther. 11, 157-168 (2011).

4. Mathew, R. et al. Autophagy suppresses tumorigenesis through elimination of p62. Cell 137, 1062-1075 (2009).

5. Yu, K. D. et al. Different distribution of breast cancer subtypes in breast ductal carcinoma in situ (DCIS), DCIS with microinvasion, and DCIS with invasion component. Ann. Surg. Oncol. 18, 1342-1348 (2011).

6. Brennan, M. E. et al. Ductal carcinoma in situ at coreneedle biopsy: meta-analysis of underestimation and predictors of invasive breast cancer. Radiology 260, 119-128(2011).

Competing interests statement

The authors declare no competing financial interests. 